\title{
Chemical inhibition of RNA viruses reveals REDD1 as a host defense factor
}

\author{
Miguel A Mata ${ }^{1,10}$, Neal Satterly ${ }^{1,10}$, Gijs A Versteeg ${ }^{2,10}$, Doug Frantz ${ }^{3}$, Shuguang Wei ${ }^{3}$, Noelle Williams ${ }^{3}$, \\ Mirco Schmolke' ${ }^{2}$, Samuel Peña-Llopis ${ }^{4-6}$, James Brugarolas ${ }^{4-6}$, Christian V Forst' ${ }^{7}$, Michael A White', \\ Adolfo García-Sastre ${ }^{2,8,9}$, Michael G Roth ${ }^{3} \&$ Beatriz M A Fontoura ${ }^{1 \star}$
}

\begin{abstract}
A chemical genetics approach was taken to identify inhibitors of NS1, a major influenza A virus virulence factor that inhibits host gene expression. A high-throughput screen of 200,000 synthetic compounds identified small molecules that reversed NS1-mediated inhibition of host gene expression. A counterscreen for suppression of influenza virus cytotoxicity identified naphthalimides that inhibited replication of influenza virus and vesicular stomatitis virus (VSV). The mechanism of action occurs through activation of REDD1 expression and concomitant inhibition of mammalian target of rapamycin complex 1 (mTORC1) via TSC1-TSC2 complex. The antiviral activity of naphthalimides was abolished in REDD1--cells. Inhibition of REDD1 expression by viruses resulted in activation of the $\mathrm{mTORC1}$ pathway. REDD1 ${ }^{-/}$cells prematurely upregulated viral proteins via mTORC1 activation and were permissive to virus replication. In contrast, cells conditionally expressing high concentrations of REDD1 downregulated the amount of viral protein. Thus, REDD1 is a new host defense factor, and chemical activation of REDD1 expression represents a potent antiviral intervention strategy.
\end{abstract}

nfections by influenza viruses are responsible for $\sim 36,000$ deaths
annually in the United States ${ }^{1}$ and $\sim 500,000$ deaths worldwide per year ${ }^{2}$. Highly pathogenic strains have been responsible for many deaths worldwide, such as the strain that caused the 1918 pandemic, which killed $\sim 30$ million people. Currently, there are only two approaches available for preventing or treating epidemic and pandemic influenza: vaccination and inhibition of virus replication. Vaccination, although highly effective against homologous strains, loses its efficacy in the elderly and is limited by the highly mutable nature of the virus and the large reservoir of antigenically distinct virus strains. These factors require the annual reformulation of the vaccine to match the antigenicity of the current circulating strains of influenza virus. A number of drugs have been approved for the treatment of influenza. These drugs inhibit virus uncoating (inhibitors of the viral protein M2) or virus spread (inhibitors of the viral protein neuraminidase), but the use of the relatively small number of such antiviral drugs is limited by the emergence of resistant virus strains. There is a clear need for additional therapeutic modalities for the treatment of disease caused by influenza virus as well as for a better understanding of mechanisms of viral-host interactions, which could lead to the discovery of new targets for therapeutic intervention.

Many viruses target host mechanisms that are key steps within pathways that regulate antiviral responses. For example, the NS1 protein of influenza virus is a multifunctional virulence factor that inhibits host gene expression and signal transduction required to induce antiviral responses. NS1 is found in both the nucleus and the cytoplasm of influenza virus-infected cells $s^{3,4}$. The cytoplasmic pool of NS1 inhibits interferon (IFN) gene induction by antagonizing the cytoplasmic signal transduction pathway mediated by RIG-I ${ }^{5-8}$. NS1 also prevents IFN action by sequestering double-stranded RNA, targeting the function of downstream antiviral effector proteins, such as the PKR and/or RNase L pathways ${ }^{9,10}$. In addition, NS1 has been shown to activate phosphatidylinositol 3-kinase (PI3K) signaling, a function that supports virus replication ${ }^{11}$.

The nuclear pool of NS1 inhibits host mRNA processing, including splicing $^{12-14}$, polyadenylation ${ }^{15}$ and nuclear export ${ }^{16-18}$, thereby preventing proper expression of host antiviral genes but not nuclear export of viral RNAs ${ }^{15,18}$. Disruption of NS1 functions by mutations yielded highly attenuated viruses that can only replicate in immunocompromised hosts ${ }^{19}$. These findings underscore the key role of NS1 as a proviral virulence factor and emphasize the need to identify its inhibitors as well as previously unknown host antiviral mechanisms that antagonize its functions. Here we performed a high-throughput screen to identify small molecules that reversed NS1-mediated inhibition of host gene expression. We identified nontoxic small molecules from the naphthalimide family that inhibited replication of evolutionarily diverse viruses, including influenza virus and VSV. We show how these small molecules serve as probes to identify the mTORC1 inhibitor REDD1 (refs. 20,21) as a new host defense factor. These findings underscore the importance of regulating REDD1 expression as a unique strategy to trigger antiviral response.

\section{RESULTS \\ Naphthalimides antagonize NS1 and influenza virus}

We exploited the potent ability of NS1 to inhibit gene expression by blocking mRNA processing and export ${ }^{15,18}$ as the basis for a high-throughput assay that measured the effect of NS1 on luciferase expression. Luciferase activity was reduced 95\% in cells transfected with plasmids encoding NS1 compared to

'Department of Cell Biology, University of Texas Southwestern Medical Center, Dallas, Texas, USA. ${ }^{2}$ Department of Microbiology, Mount Sinai School of Medicine, New York, New York, USA. ${ }^{3}$ Department of Biochemistry, University of Texas Southwestern Medical Center, Dallas, Texas, USA. ${ }^{4}$ Department of Internal Medicine, University of Texas Southwestern Medical Center, Dallas, Texas, USA. ${ }^{5}$ Department of Developmental Biology, University of Texas Southwestern Medical Center, Dallas, Texas, USA. ${ }^{\circ}$ Simmons Cancer Center, University of Texas Southwestern Medical Center, Dallas, Texas, USA. ${ }^{7}$ Department of Clinical Sciences, University of Texas Southwestern Medical Center, Dallas, Texas, USA. ${ }^{8}$ Department of Medicine, Division of Infectious Diseases, Mount Sinai School of Medicine, New York, New York, USA. ${ }^{9}$ Global Health and Emerging Pathogens Institute, Mount Sinai School of Medicine, New York, New York, USA. ${ }^{10}$ These authors equally contributed to the manuscript. *e-mail: beatriz.fontoura@utsouthwestern.edu 


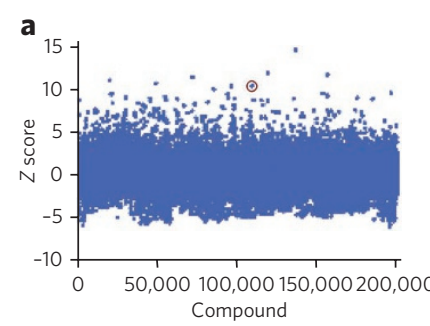

b

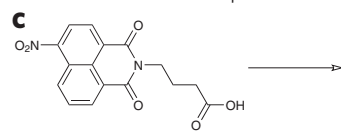

(1)

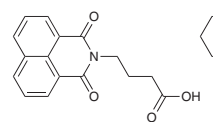

(2)

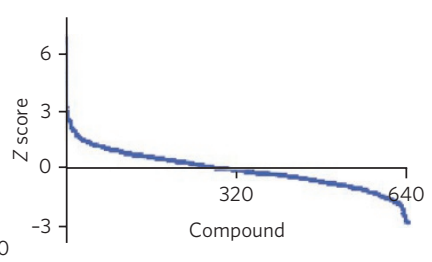

Figure 1 | Identification of small molecules that revert the inhibition of gene expression mediated by the influenza virus NS1 protein and protect cells from virus-induced cell death. (a) Luciferase expression in HEK 293T cells transfected with NS1 and treated individually with 200,000 synthetic compounds ( $5 \mu \mathrm{M}$ ) was normalized to values for on-plate controls treated with $0.3 \%$ DMSO. Values are expressed as $Z$ scores using the mean value and s.d. of the experimental population screened on the same day. Red circle shows compound $\mathbf{1}$ studied here. (b) Inhibition of influenza virus-mediated cell death. The 640 most active compounds were tested at three concentrations for the ability to inhibit the cytopathic effect of A/WSN/1933 influenza virus infection in HBECs. Z scores for compounds assayed at $1.7 \mu \mathrm{M}$ are plotted according to activity. (c) The structure of the most active naphthalimide from the primary screen (1), an inactive analog (2) and a more potent related compound (3) are shown.

cells expressing luciferase alone, as we previously reported ${ }^{18}$. We screened 200,000 compounds at 5- $\mu \mathrm{M}$ concentrations with this assay and then counterscreened for the ability of these small molecules to suppress cytotoxicity caused by influenza virus infection
(Fig. 1a,b). Among the most active compounds was 4-[N-4-nitro(1,8-naphthalimide)]-butanoic acid, compound 1 (Fig. 1c).

We obtained compounds structurally related to 1 and identified some that had no antiviral activity (2) or more potent activity (3) (Fig. 1c, Supplementary Methods, Supplementary Results, Supplementary Fig. 1). Compound 3 was much less cytotoxic (Fig. 2a) and had a much longer half-life than the original compound (1) (Fig. 2b). Compound 3 also did not alter bulk protein synthesis (Supplementary Fig. 2). To investigate the effect of $\mathbf{3}$ on virus-mediated cytotoxicity, we infected MDCK cells with influenza A/WSN/1933 virus at a multiplicity of infection (m.o.i.) of 0.001 for $48 \mathrm{~h}$ in the presence or absence of $\mathbf{3}$. Widespread cytopathic effects were observed in MDCK cells in the absence of 3 after $48 \mathrm{~h}$ of infection, but 3 largely prevented this effect (Fig. 2c). Because this compound was derived from a screen in which we observed a reversal of NS1-mediated inhibition of gene expression, we expected that a significant reversal of the mRNA-export block induced by influenza virus would occur in the presence of active compound. Indeed, in cell populations infected with influenza virus in the presence of $\mathbf{3}$, there was a decrease in the number of cells that retained poly(A) RNA in the nucleus compared to the number of infected cells not treated with $\mathbf{3}$ (Fig. 2d,e). A subpopulation of infected cells still presented mRNAexport block in the presence of 3 ; thus, it is possible that these cells are at different phases of the cell cycle, which is known to regulate mRNA export ${ }^{22}$. Thus, 3 partially antagonizes the mRNA export block in virus-infected cells.

\section{Naphthalimide inhibits virus replication}

The effect of 3 on virus replication was then assessed using various strains of influenza virus: A/WSN/1933, A/Texas/1991 and the highly virulent A/Brevig/Mission/1/1918 strain that killed $\sim 30$ million people ${ }^{23}($ Fig. 3a-c). Noncytotoxic concentrations of 3 reduced viral
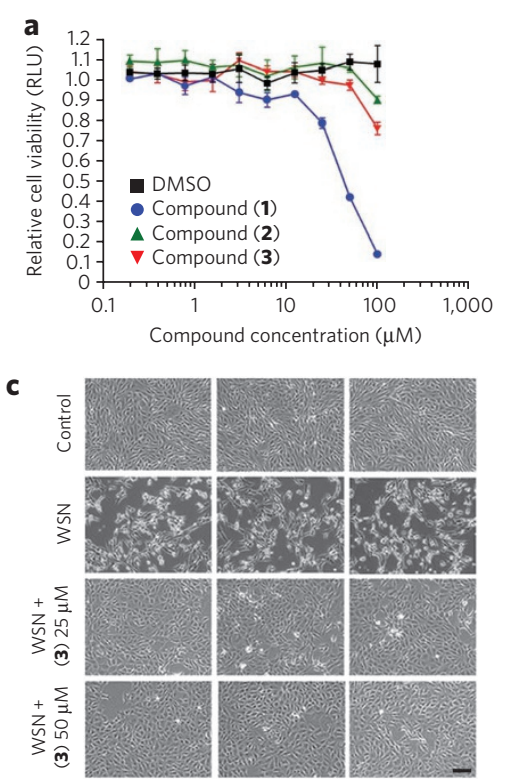
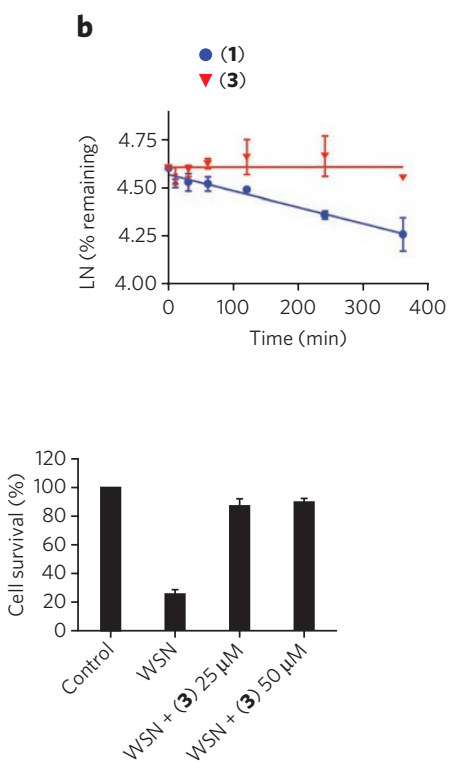
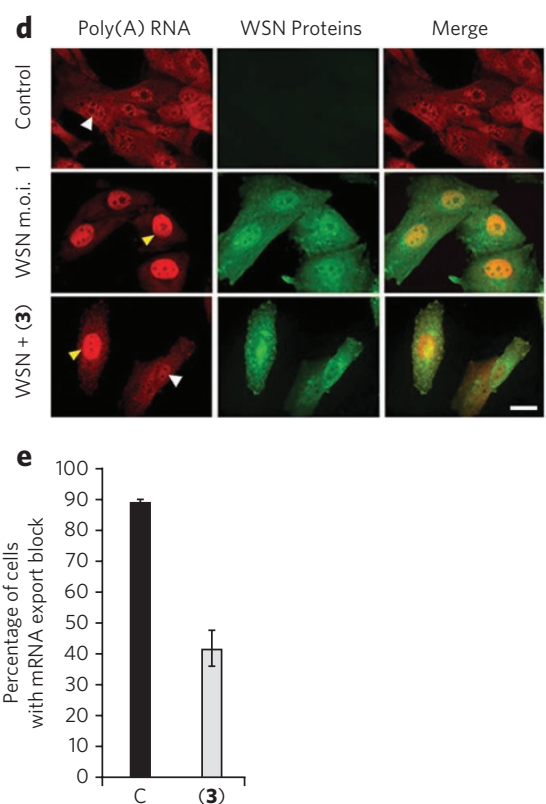

Figure 2 | Compound 3 is less cytotoxic, more stable than 1 and reverts influenza virus-mediated cytotoxicity and mRNA export block. (a) MDCK cells were treated for $30 \mathrm{~h}$ with compounds $\mathbf{1}, \mathbf{2}$ and $\mathbf{3}$ at the various concentrations depicted, and control cells were treated with the same concentration of DMSO as those in the wells containing compound. Cell viability was determined by measuring cell ATP concentrations. RLU, relative light units. (b) The fraction of compound remaining in cells treated with $\mathbf{1}$ or $\mathbf{3}$ as a function of incubation time was determined by mass spectrometry. LN, natural log. (c) MDCK cells were pretreated for $17 \mathrm{~h}$ with DMSO or with the indicated concentrations of $\mathbf{3}$ and subsequently mock infected or infected with A/WSN/1933 virus at m.0.i. 0.001 for $48 \mathrm{~h}$. The indicated concentrations of compound were present during infection. Differential interference contrast (DIC) microscopy imaging was performed in a Zeiss Axiovert $200 \mathrm{M}$. Cell survival was determined by counting live cells. Scale bar, $90 \mu \mathrm{m}$. (d) MDCK cells, mock infected or infected with A/WSN/1933 in the presence or absence of $25 \mu \mathrm{M}$ 3, were fixed and subjected to oligo-dT in situ hybridization to detect poly(A) RNA distribution in the nucleus and cytoplasm. Influenza proteins were detected by immunofluorescence using antibodies specific for influenza proteins. Yellow arrowheads point to cells with mRNA export block, whereas white arrowheads point to cells that do not show blockage. Scale bar, $15 \mu \mathrm{m}$. (e) Data from triplicate experiments as depicted in $\mathbf{d}$ were quantified and the percentage of infected cells retaining mRNA in the nucleus is shown. Data represent mean values \pm s.d. 


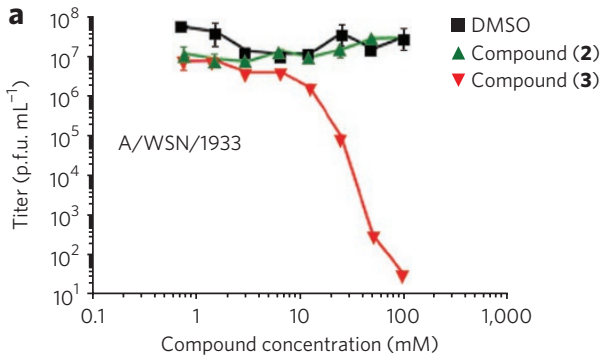

d

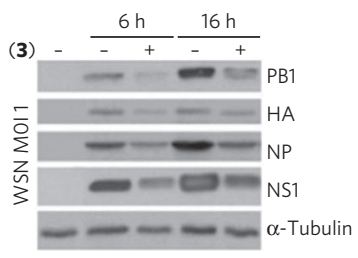

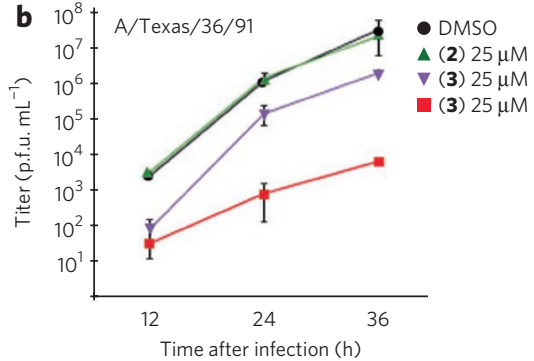

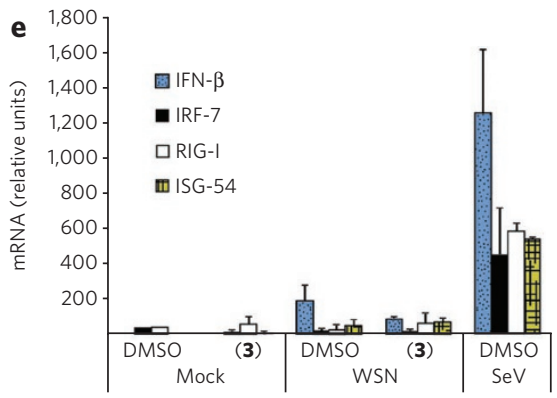

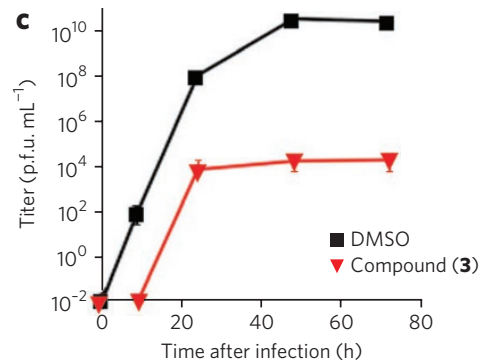

f

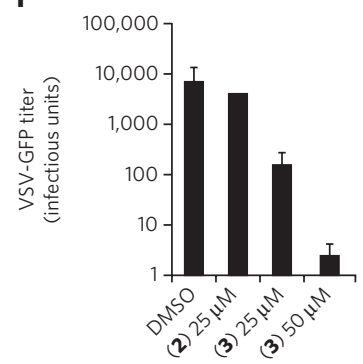

Figure 3 | Compound $\mathbf{3}$ inhibits virus replication but does not induce IFN response. (a-c) MDCK cells mock infected or infected at m.0.i. 0.001 with the influenza virus strains shown were left untreated or treated with compounds at the depicted concentrations, and the virus titers of culture supernatants were determined by plaque assay. Strain A/WSN/1933 is in $\mathbf{a}, A / T e x a s / 36 / 91$ is in $\mathbf{b}$ and A/Brevig/Mission/1/1918 is in c. (d) Intracellular viral protein concentrations were measured by immunoblot analysis with specific antibodies to the indicated proteins. (e) Human A549 cells treated with DMSO or $25 \mu \mathrm{M} 3$ were mock infected or infected with A/WSN/1933 at m.o.i. 0.001, and after 36 h, RNA was isolated and the expression of the IFN-responsive genes shown was quantified by real-time PCR. (f) MDCK cells mock infected or infected with VSV-GFP (m.o.i. $=0.001)$ were untreated or treated with the indicated compounds. At $24 \mathrm{~h}$ after infection, virus titers were determined in the supernatants. Data represent mean values $\pm \mathrm{s}$.d.

titers by $10^{3}$ to $10^{6}$ between 24 to $36 \mathrm{~h}$ after infection, depending on the influenza virus strain. The ratio of the concentration causing half-maximum cytotoxicity $\left(\mathrm{CC}_{50}\right)$ to half-maximum inhibitory concentration $\left(\mathrm{IC}_{50}\right.$ ) for $\mathbf{3}$ was 31 (Figs. 2a and 3a). Similar results were also observed in human A549 cells (Supplementary Fig. 3). As shown in Figure 3d, intracellular influenza virus proteins were also downregulated in the presence of $\mathbf{3}$. Thus, $\mathbf{3}$ decreased viral protein levels, contributing to the reduction of virus replication.

The antiviral effect of $\mathbf{3}$ was not mediated by IFN. The mRNA levels of IFN- $\beta$ and IFN effectors were measured by quantitative PCR and microarray analysis, and the results revealed that 3 did not induce IFN production or an IFN-mediated response (Fig. $3 \mathbf{e}$ and Supplementary Data Set 1). Furthermore, cells that had impaired IFN response, Vero cells and Stat $1^{-/}$cells, were protected by 3 from influenza virus replication and cell death, respectively (Supplementary Fig. 4). Compound 3 also antagonized expression of large amounts of influenza virus proteins in Vero cells (Supplementary Fig. 5). Thus, 3 partially antagonized the block of mRNA export in virusinfected cells, but this effect did not result in the production of IFN. However, the partial relief of mRNA-export inhibition by $\mathbf{3}$ was probably a consequence of low NS1 concentrations, which resulted in the expression of host mRNAs that encode antiviral factors. To investigate whether $\mathbf{3}$ antagonized NS1 directly or promoted host antiviral functions regulated by NS1 that could also affect replication of other viruses, we infected cells with VSV at 0.001 plaque-forming units (p.f.u.) per cell in the absence or presence of $\mathbf{2}$ or $\mathbf{3}$. Compound 3 inhibited VSV replication (Fig. 3f). Thus, 3 targets host cell functions that confer an antiviral state against diverse viruses.

\section{Antiviral activity of naphthalimide requires REDD1}

Because 3 targeted the host, we analyzed host pathways by comparing gene expression profiles in human A549 cells in the presence or absence of compound using gene set enrichment analysis (Supplementary Fig. 6, Supplementary Data Set 1 and Supplementary Methods). In cells treated with 3 , the mTORC1 pathway had one of the highest enrichment scores. REDD1, an inhibitor of the mTORC1 pathway ${ }^{20,21}$, was upregulated at the mRNA level (Fig. 4a and Supplementary Data Set 1). The induction of REDD1 mRNA by 3 was abolished in the presence of the transcription inhibitor actinomycin D (Fig. 4a). In addition, REDD1 mRNA decayed over time in the absence or presence of 3 and actinomycin D (Fig. 4a). Thus, these results indicate that induction of REDD1 mRNA by 3 occurs at the transcriptional level. REDD1 protein levels increased approximately six- to eightfold in the presence of $\mathbf{3}$ alone or in the presence of both $\mathbf{3}$ and influenza virus infection (Fig. 4b). Again, this induction of REDD1 protein by 3 was abolished in the presence of actinomycin D (Fig. 4b). We found that influenza virus greatly increased the degree of phosphorylation on S6 kinase (p70-S6K) at Thr389 (Fig. 4c), a site phosphorylated by mTORC1, and this effect was greatly reduced in A549 cells treated with 3 (Fig. 4c). That the total amount of S6K protein did not change in the presence of $\mathbf{3}$ (Fig. $\mathbf{4 c}$ ) demonstrates that the effect of this small molecule occurred at the level of phosphorylation of S6K at Thr389. The mTORC1 inhibitor rapamycin also reduced the amount of influenza virus NS1 protein (Supplementary Fig. 7).

To investigate whether 3 prevented S6K activation independently of influenza virus, we tested the effect of 3 in H358 non-small cell lung cancer cells, which have chronically active S6K. Cells were treated with 3 and inactive 2 , and $\mathbf{3}$, but not $\mathbf{2}$, reduced the activation of S6K in H358 cells (Supplementary Fig. 8a). In two other cancer cell lines with chronically active AKT, H1993 and LnCAP, 3 also reduced the activation of S6K (Supplementary Fig. 8b). However, 3 did not inhibit phosphorylation of a major active site, Ser473, on AKT (Fig. 4d), which is a target of mTORC2 (refs. 24,25). In A549 cells infected with influenza virus for $7 \mathrm{~h}, 3$ blocked S6K activation and had no effect on AKT phosphorylation (Fig. 4d). At $22 \mathrm{~h}$ after infection, 3 did not alter phosphorylation at AKT Thr308 but reduced phosphorylation at AKT Ser473; however, this reduction is probably an indirect effect of $\mathbf{3}$ on the inhibition of viral replication rather than a direct effect of $\mathbf{3}$ on AKT. Thus, $\mathbf{3}$ acts in parallel to or downstream of AKT. 


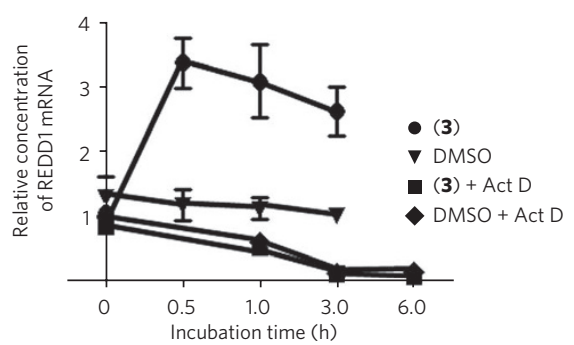

b

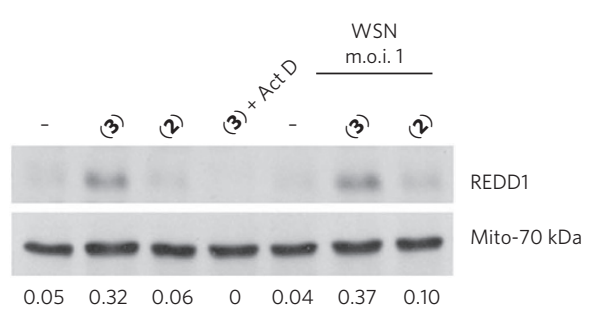

c
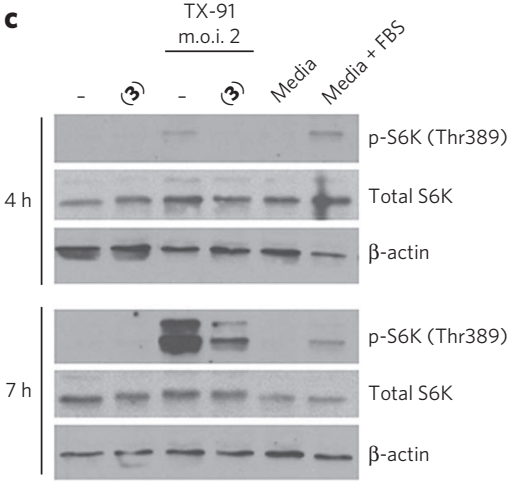

d

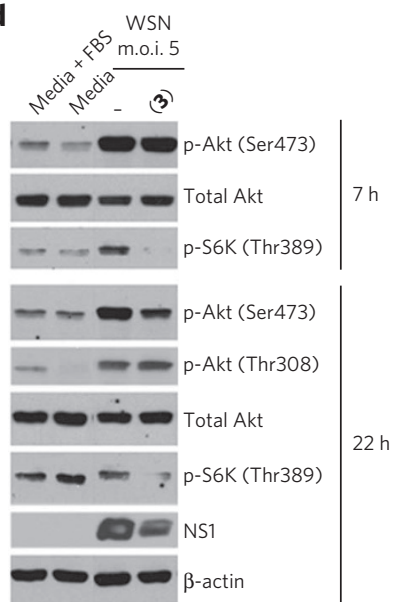

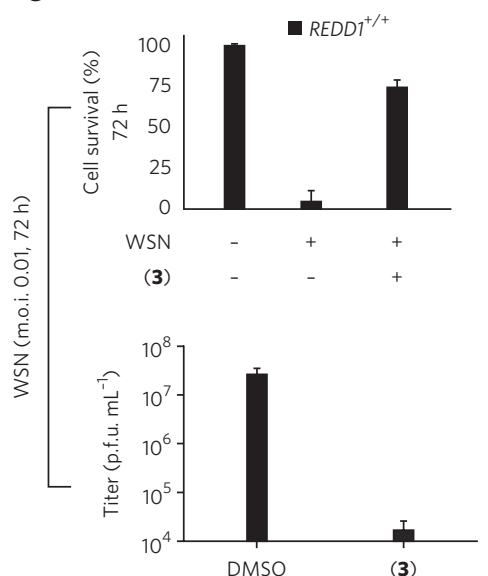

f

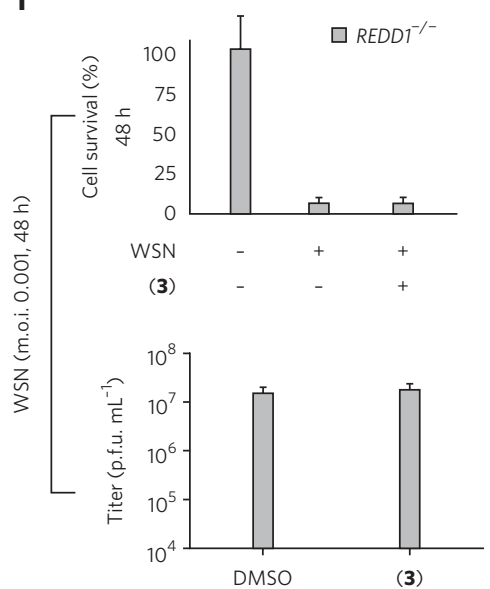

Figure 4 | Influenza virus activates the mTORC1 pathway and naphthalimide requires the mTORC1 inhibitor REDD1 for its antiviral activity. (a) A549 cells were untreated or treated with $30 \mu \mathrm{M} \mathbf{3}$ for the indicated time periods, in the absence or presence of actinomycin D $\left(0.5 \mu \mathrm{g} \mathrm{ml} \mathrm{l}^{-1}\right)$. REDD1 mRNA levels were quantified by real time PCR. (b) A549 cells were untreated or treated with $30 \mu \mathrm{M} \mathbf{3}$ (in the absence or presence of $0.5 \mu \mathrm{g} \mathrm{ml}{ }^{-1}$ actinomycin $\mathrm{D}$ as indicated) for $18 \mathrm{~h}$ before infection and during infection. Cell extracts were obtained at $6 \mathrm{~h}$ after infection and subjected to immunoblot analysis with the indicated antibodies. Densitometry analysis was performed to determine the ratio of REDD1 over loading control (Mito-70 kDa) using ImageJ (Supplementary Fig. 18). (c) A549 cells were untreated or treated as in b before and during infection. Cell extracts were subjected to immunoblot analysis with depicted antibodies (Supplementary Fig. 19). (d) Phosphorylation of Akt or S6K was measured by immunoblot analysis in cell extracts of A549 cells infected with influenza virus in the presence or absence of 3. Compound was added prior to and during infection as in b (Supplementary Fig. 20). $(\mathbf{e}, \mathbf{f}) R E D D 1^{+/+}$cells were untreated or treated with $\mathbf{3}$ and mock-infected or infected at m.o.i 0.01 with A/WSN/1933 for $72 \mathrm{~h}(\mathbf{e}) . R E D D 1^{-/-}$MEF cells, untreated or treated with 3, were infected with A/WSN/1933 at m.o.i. 0.001 for $48 \mathrm{~h}$ (f). Cell survival was determined by Trypan blue exclusion assay and virus titers were measured by plaque assays. Data represent mean values \pm s.d.

To determine whether REDD1 was required for the antiviral activity of $\mathbf{3}$, we tested the antiviral effect of $\mathbf{3}$ in infected $R E D D 1^{+/+}$ or $R E D D 1^{-1}$ mouse embryonic fibroblasts. Influenza virus-mediated cell death and replication were inhibited by 3 in $R E D D 1^{+/+}$cells infected at m.o.i. 0.01 for $72 \mathrm{~h}$ (Fig. 4e); we infected the cells at $72 \mathrm{~h}$ because, at this point, enough cell death had occurred so that we could determine protection by 3 . Infected $R E D D 1^{-1}$ cells treated in the same conditions as $R E D D 1^{+/+}$cells were completely dead by
$24 \mathrm{~h}$ in the presence or absence of compound; therefore, we infected $R E D D 1^{-/}$cells with influenza virus at m.o.i. 0.001 for $48 \mathrm{~h}$, in the absence or presence of 3 . Even with this low m.o.i. and short infection time, 3 did not protect $R E D D 1^{--}$cells from virus-mediated cell death or virus replication (Fig. $\mathbf{4 f}$ ). In addition, $R E D D 1^{-1}$ cells infected at m.o.i. 0.001 for $48 \mathrm{~h}$ produced approximately as many viral particles as $R E D D 1^{+/+}$cells infected at m.o.i. 0.01 for $72 \mathrm{~h}$ (Fig. 4e,f). When $R E D D 1^{-/}$cells were infected with influenza virus at m.o.i. 0.001, a

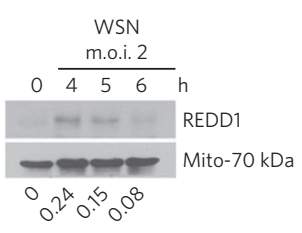

b

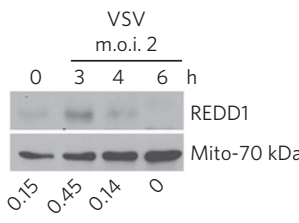

c

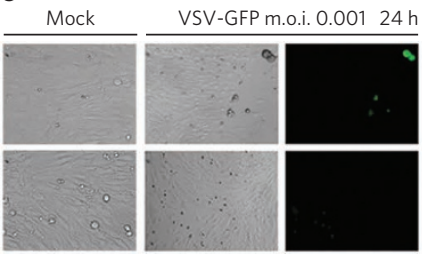

$\operatorname{REDDD}^{-1-}$

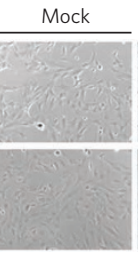

VSV-GFP m.o.i. $0.00124 \mathrm{~h}$

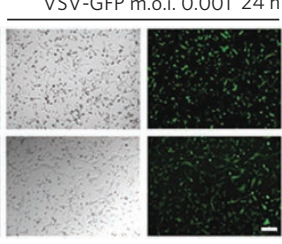

d

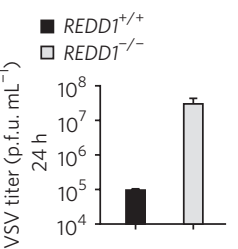

Figure $\mathbf{5}$ | Viruses activate the mTORC1 pathway via downregulation of REDD1 expression. Extracts from cells mock infected or infected with influenza virus (a) or VSV-GFP (b) were subjected to immunoblot analysis with depicted antibodies. Densitometry analysis was performed to determine the ratio of REDD1 over loading control (Mito-70 kDa) using ImageJ. (c) Wild-type or REDD1-/ MEF cells were infected with VSV-GFP at m.o.i. of 0.001 for $24 \mathrm{~h}$. DIC or fluorescent images of VSV-GFP are shown. Scale bar, $50 \mu \mathrm{m}$. (d) Supernatants of cells from c were subjected to plaque assays. Data represent mean values \pm s.d. 


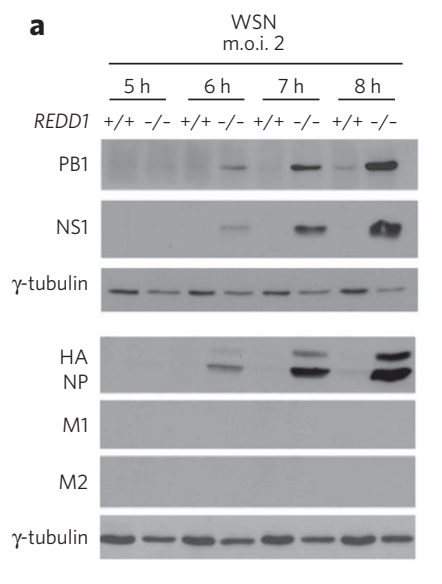

WSN
m.o.i. 2

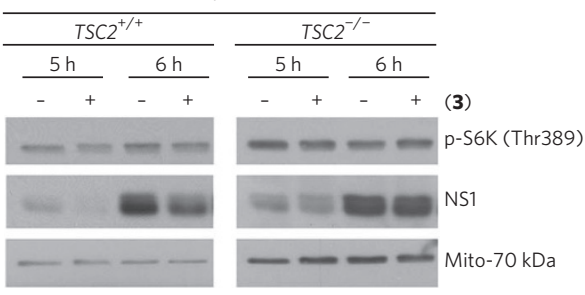

b
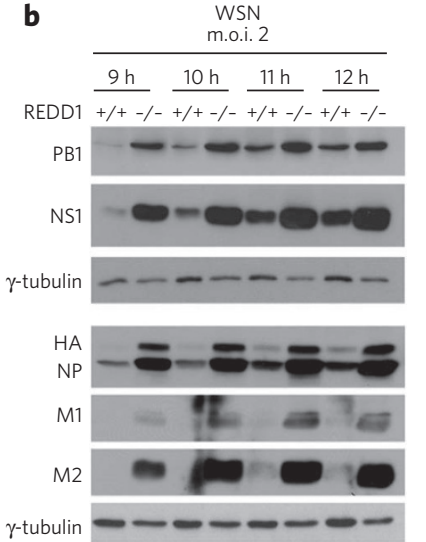

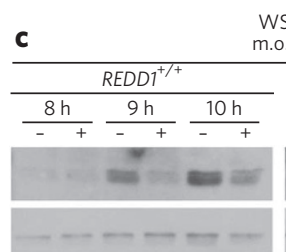

WSN
m.o.i. 1

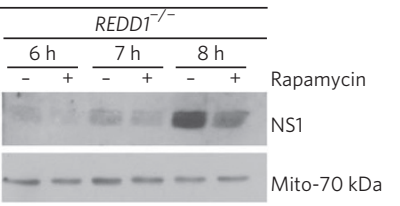

d
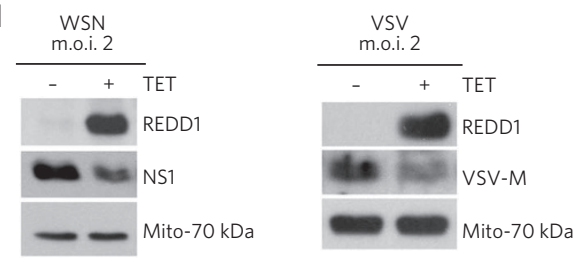

f

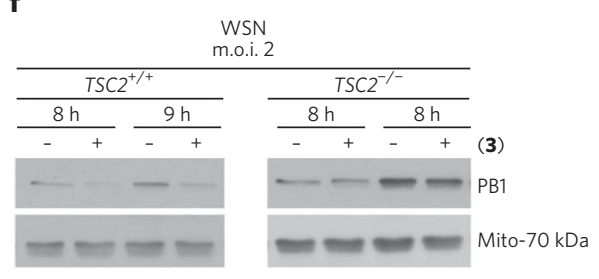

Figure 6 | REDD1 regulates viral protein expression in an mTORC1-dependent manner. (a) $R E D D 1^{+/+}$and $R E D D 1^{-/-}$cells were infected with influenza virus WSN at m.o.i. 2 for $1 \mathrm{~h}$ at $22^{\circ} \mathrm{C}$ and then shifted to $37^{\circ} \mathrm{C}$. Viral protein levels were monitored over time by immunoblot analysis with the depicted antibodies. (b) Viral protein levels were monitored as in a. (c) WSN-infected REDD1+/+ and REDD1-/- cells were treated with $100 \mathrm{nM}$ rapamycin. Rapamycin was added $1 \mathrm{~h}$ after infection. NS1 levels were monitored over time by immunoblot analysis. (d) U2OS cells, untreated or treated with tetracycline to induce REDD1 overexpression, were infected as in a but with both influenza virus and VSV. NS1 or VSV-M protein levels were monitored by immunoblot analysis. (e,f) $\mathrm{TSC2}^{+/+}$and $\mathrm{TSC}^{--}$cells were pretreated with $10 \mu \mathrm{M}$ 3. Cells were then infected with influenza virus WSN at $\mathrm{m} .0 . i .2$ for $1 \mathrm{~h}$ at $22{ }^{\circ} \mathrm{C}$ and then shifted to $37^{\circ} \mathrm{C}$ in the absence of compound. After $1 \mathrm{~h}$ of infection, $\mathbf{3}$ was added back. Cell extracts were obtained at $5 \mathrm{~h}$ and $6 \mathrm{~h}$ after infection in (e) and $8 \mathrm{~h}$ and $9 \mathrm{~h}$ after infection in (f), then subjected to immunoblot analysis with the indicated antibodies.

they produced $\sim 200$-fold more virus than $R E D D 1^{+/+}$cells infected in the same conditions (Supplementary Fig. 9). This effect was also observed in VSV-infected REDD1 $1^{+/+}$and $R E D D 1^{-/-}$cells (described further below). Treatment of both $R E D D 1^{+/+}$and $R E D D 1^{-/}$cells with 3 alone did not cause cytotoxicity (Supplementary Fig. 10). Thus, REDD1 knockout cells were more permissive to influenza virus replication than wild-type cells. As 3 did not inhibit virus replication in the absence of REDD1 (Fig. 4f), REDD1 is required for the antiviral activity of $\mathbf{3}$.

\section{REDD1 is a host defense factor}

These data indicate that REDD1 is an important host factor required for antiviral response, raising the possibility that viruses regulate REDD1 expression. During influenza virus and VSV infections, REDD1 expression initially increased but was then downregulated (Fig. 5a,b), resulting in activation of S6K (Fig. 4c). The initial upregulation of REDD1 probably represented a host antiviral response, which was then inhibited by the virus, resulting in activation of mTORC1. Consistent with REDD1's involvement in a general host-cell antiviral response, $R E D D 1^{-/}$cells were also highly permissive to VSV replication compared to wild-type cells (Fig. 5c,d), resulting in higher levels of intracellular VSV proteins in $R E D D 1^{-/-}$cells than in $R E D D 1^{+/+}$cells (Supplementary Fig. 11). That 3 did not inhibit VSV replication in the absence of REDD1, as it did in $R E D D 1^{+/+}$cells, shows once again that REDD1 is required for its antiviral activity (Supplementary Fig. 12).

By preventing viruses from activating mTORC1, REDD1 might affect two biological functions potentially important for virus replication: autophagy or protein translation. By preventing activation of mTORC1, enhanced REDD1 expression may increase autophagy ${ }^{26}$. However, compound 3 protected $A T G 5^{-1}$ cells, which lack an autophagic response, against VSV replication (Supplementary Fig. 12). In addition, treatment of cells with chloroquine, an autophagy inhibitor, did not affect the amount of viral protein in $R E D D 1^{--}$cells (Supplementary Fig. 13). Together, these results indicate that autophagy was not the mechanism involved in 3-mediated inhibition of viral protein expression. Thus, the requirement for activating mTORC1 for efficient virus replication is likely to be translation.

To determine whether the enhanced viral infection in $R E D D 1^{-1-}$ cells was due to a general increase in translation or an effect on specific viral proteins, we measured the expression of several influenza virus proteins as a function of time after infection of both REDD1 wildtype and knockout cells. We subjected lysates from $R E D D 1^{+/+}$and $R E D D 1^{-/}$cells infected with influenza virus to immunoblot analysis with antibodies against various influenza virus proteins. $R E D D 1^{-/}$ cells produced large amounts of influenza virus proteins $2-3 \mathrm{~h}$ earlier than $R E D D 1^{+/+}$cells (Fig. 6a,b). The enhanced expression of viral proteins led to increased viral RNA levels (Supplementary Fig. 14), and we observed similar results upon VSV infection (Supplementary Fig. 11). To determine whether the effect on viral proteins in $R E D D 1^{-1-}$ cells was due to the activity of mTORC1 in translation, we treated $R E D D 1^{--}$cells with rapamycin. In fact, the downregulation of viral protein caused by rapamycin treatment in both $R E D D 1^{+/+}$and $R E D D 1^{-/}$cells (Fig. 6c) (Supplementary Fig. 15) indicated that induction of high viral protein levels in $R E D D 1^{--}$cells occurs via activation of mTORC1. Furthermore, in cells conditionally expressing large amounts of REDD1, the concentration of viral protein was reduced, consistent with the function of REDD1 as a host defense factor (Fig. 6d). 
REDD1 prevents the inactivation of the TSC1-TSC2 complex by AKT1 and, thus, blocks activation of the mTORC1 pathway ${ }^{20,27}$. In TSC2-knockout cells, 3 did not induce downregulation of viral protein expression, as opposed to wild-type cells in which viral protein levels were inhibited by 3 (Fig. 6e,f). In addition, activation of S6K in $R E D D 1^{-/}$-infected cells was not inhibited by 3 (Supplementary Fig. 16), indicating that $\mathbf{3}$ does not act directly on S6K. Thus, 3 requires TSC2 for downregulating viral protein expression (Fig. 6e,f). Altogether, these findings show that the antiviral activity of 3 occurs by repressing the activity of mTORC1 in a TSC1-TSC2-dependent manner. It is also possible that $\mathbf{3}$ may act on other pathways.

We also designed an analog of 3, termed 4, which has similar antiviral properties to 3 . Compound 4 prevented virus replication by inducing REDD1 but was a more potent inhibitor of the highly pathogenic H1N1/1918 influenza virus strain (Supplementary Fig. 17) ${ }^{23}$. Altogether, these findings reveal REDD1 as a new host antiviral factor and show that the antiviral activity of 3 requires REDD1.

\section{DISCUSSION}

There are essentially two approaches for identifying unique host processes that are involved in antiviral functions and can be exploited therapeutically. One is to learn as much as possible about the host mechanisms required by the virus and then test the effects of inhibiting them. The other is to take an unbiased approach and screen for chemical inhibitors of virus functions or host genes required by the virus. Taking the chemical-genetics version of the second approach, we conducted a screen for compounds that antagonized the inhibition of gene expression by NS1 and identified napthalimides that inhibited replication of influenza viruses and VSV. These compounds functioned by increasing expression of REDD1, a major negative regulator of the mTORC1 pathway, and in cells lacking REDD1 the compound lost its antiviral activity.

Many viruses activate AKT by stimulating PI3K ${ }^{28,29}$. The direct binding of NS1 protein of influenza virus to PI3K results in activation of $\mathrm{AKT}^{30-33}$. This has been interpreted either as a means to inhibit apoptosis and prevent the cell from dying prematurely during infection or as a necessary step in promoting virus replication. A recent genome-wide siRNA screen implicated mTORC1 in influenza virus replication $^{34}$, suggesting that activation of that pathway might be one of the functions of elevated AKT1 signaling. Our results imply that a major consequence of AKT signaling for influenza virus replication is activation of the mTORC1 effector S6K through phosphorylation, as the antiviral napthalimides we identified inhibited phosphorylation of S6K by mTORC1. We showed that the protein upregulated by our napthalimides, the mTORC1 inhibitor REDD1, is a new host defense factor. Its production was at first induced by influenza virus or VSV but was then successfully suppressed by the virus. REDD1 suppression by viruses promoted virus replication, as REDD1-knockout cells were highly permissive to virus replication.

REDD1 is induced by various environmental conditions, including cell confluency, glucocorticoid treatment, hypoxia and other stress-response pathways such as endoplasmic reticulum (ER) stress $^{35}$. Both ER stress and hypoxia-inducible factors (HIFs) have a role in immunity and infection ${ }^{36,37}$. ER stress has been shown to promote plasma-cell development, and the absence of key components in this pathway results in sensitization to viral infection ${ }^{36}$. Mouse embryonic fibroblasts deficient in the ER protein kinase PERK, which is activated by accumulated unfolded proteins in the $\mathrm{ER}$, are more permissive to VSV replication than wild-type cells ${ }^{38}$. Upregulation of REDD1 in response to ER stress ${ }^{39,40}$ occurs via the transcription factor ATF4 (ref. 40). HIF activation by the hypoxia mimetic cobalt chloride promotes cellular resistance to VSV infection, whereas HIF inhibition by RNA interference or a smallmolecule antagonist has shown increased sensitivity to viral infection, as measured by enhanced VSV cytotoxicity and replication ${ }^{37}$; however, the mechanism is not known. During hypoxia, REDD1 has been shown to be a direct target of the HIF-1 $\alpha$ transcription factor $^{20}$, which induces REDD1 expression. Thus, activating a stressresponse pathway or promoting the expression of a stress-response protein may to a certain extent induce resistance to pathogens and decrease host cytotoxicity. However, the coordination of a stress response to promote cellular resistance without marked damage to the host upon pathogen invasion remains to be further investigated. We showed that induction of REDD1 by small molecules is an efficient strategy for interfering with the functions of the mTORC1 pathway that are required by viruses.

The effect of napthalimide on influenza virus was a sharp attenuation of the production of virus proteins early in infection. We found no effect of the napthalimide on global protein synthesis and no induction of an IFN response. In addition, in cells lacking REDD1, in which expression of influenza virus proteins is enhanced, rapamycin inhibited expression of influenza virus proteins at a concentration that is known not to alter bulk protein synthesis. This indicates selective translational regulation, which has been documented in a number of conditions, including the general amino acid control response $^{41}$ and other types of processes such as survival or proliferation $^{42}$. In addition, during nuclear mRNA processing and export, specific sequences within either the untranslated regions or the coding region can dictate the differential binding of RNA-binding proteins (specifically, heterogeneous ribonucleoprotein particles), which will in turn regulate processing and export of specific subsets of mRNAs to result in differential expression ${ }^{43,44}$. This raises the possibility that the inhibition of the mTORC1 pathway may alter translation in a way that is unfavorable to the initiation of specific viral mRNA expression, relative to that of host mRNA. In cells infected at a low m.o.i., the first viral messages must compete with the far larger volume of host messages for access to ribosomes. In this respect, the early viral messages would encounter the same problems as a hostcell message with low abundance, such as mRNAs encoding certain transcription factors. However, at the earliest phases of infection, viruses are largely dependent upon normal host processes, and these processes are the most likely to be the useful therapeutic targets.

Although many viruses can be controlled by vaccination, there is still an important need for antiviral drugs. For viruses that can infect other animals, such as influenza, vaccination will never lead to full eradication. Other human viruses, such as smallpox or measles, can potentially be eradicated by global immunization, but, once the incidence of such diseases becomes very low, global vaccination is inevitably discontinued, leaving the human population vulnerable to reemergence of the virus. The long lead times required to produce sufficient vaccine to protect the human population means that the appearance of a new or reoccurring highly infectious virus can lead to a pandemic of the disease before the vaccine is available, which means that antiviral drugs can be a key boon at such a time. However, antiviral drugs that target viral proteins have the disadvantage that resistance to the drug will arise because of the high rates of mutation inherent in viruses and the large numbers of progeny that they produce. A strategy targeting host processes that are essential for virus replication, such as the one discussed here, avoids this problem, although it is limited by the possibility of toxic side effects. Thus, combinations of noncytotoxic small molecules that target both viral and host proteins are desirable. Recently, influenza A nucleoprotein was identified as an antiviral target ${ }^{45}$, and a small molecule that triggered its aggregation and prevented its import into the nucleus protected against influenza virus replication ${ }^{45}$. In addition, a chemical compound that inhibited host pyrimidine biosynthesis has been recently shown to reduce influenza virus replication ${ }^{46}$.

In sum, the strategy of chemically inducing host antiviral activities that target host pathways without causing considerable short-term toxic effects will probably have a major impact on antiviral therapy. One such strategy was identified here with the induction of the 
mTORC1 inhibitor REDD1 by naphthalimides. Furthermore, small molecules that inhibit the mTORC1 pathway in different ways have the potential for anticancer therapy, as the mTORC1 pathway is a major regulator of cell proliferation and cancer ${ }^{47}$.

\section{METHODS}

Compound screen. The UT Southwestern Compound Library is composed of 200,000 synthetic-drug-like compounds arrayed in DMSO in 384 well plates. HEK 293T cells were transfected with an approximately 10:1 ratio of plasmid pCMV-Luc encoding luciferase and pCAGGS-NS1 encoding NS1 using Lipofectamine2000 (Invitrogen). Cells were transfected with the luciferase plasmid alone as a positive control. After $16 \mathrm{~h}$, cells were dispensed at 5,000 cells per well in 384-well plates. After $1 \mathrm{~h}$, compounds from the library were added to a final concentration of $5 \mu \mathrm{M}$ in $1 \%(\mathrm{v} / \mathrm{v})$ DMSO in a one compound per well format. Experimental samples were limited to columns 3 to 22, with controls treated with $1 \%$ DMSO in the first and last two columns of wells. Wells in the first column of each plate contained cells transfected with the luciferase plasmid alone; all other wells received cells transfected with both plasmids. Plates were incubated for $22 \mathrm{~h}$ at $37^{\circ} \mathrm{C}$ in $5 \% \mathrm{CO}_{2}$, then cooled to $22^{\circ} \mathrm{C}$, incubated with Bright-Glo luciferase substrate (Promega) for $4 \mathrm{~min}$, then luminescence was recorded. Experiments producing plates with standard $(Z)$ scores lower than 0.45 were repeated. Experimental values were normalized to the mean of the luciferase-only control on the same plate. Compounds were ranked by $Z$ score, and the $640 \mathrm{com}-$ pounds with the most positive $Z$ scores were selected and retested in the assay at concentrations of $15 \mu \mathrm{M}, 5 \mu \mathrm{M}$ and $1.7 \mu \mathrm{M}$. These compounds were also tested for the ability to prevent cell death of immortalized human bronchial epithelial cells (HBECs) that had been infected with A/WS/33 influenza virus by measuring cell ATP levels with ATP-lite (PerkinElmer). A table describing this screen can be found in Supplementary Table 1.

Compound half-life. Compound half-lives were measured in HBECs by LC/MS/MS. Metabolic stability half-life was determined by substrate depletion ${ }^{48}$

Cell survival and cytotoxicity measurements. MEFs, HBECs or MDCK cells were seeded in white-walled 96-well plates at a density of $3 \times 10^{3}$ cells per well, $16 \mathrm{~h}$ before compound addition. Compounds dissolved in sterile DMSO (Sigma) at a concentration of $25 \mathrm{mM}$ were diluted to $100 \mu \mathrm{M}$ in OptiMEM I (Invitrogen) in triplicates. The $100-\mu \mathrm{M}$ starting dilutions were serially diluted in twofold steps to a final concentration of $0.2 \mu \mathrm{M}$. Control experiments, performed in the absence of compound, had the same final concentration of DMSO as compound-treated samples. At the time points depicted in the figures $(24 \mathrm{~h}, 48 \mathrm{~h}$ and $72 \mathrm{~h}$ ), cells were lysed, and ATP levels were measured by luminescence using the Cell Titer-Glo kit (Promega), following manufacturer instructions. In parallel, cells were also counted at the beginning and at the end of each experiment, and cell survival was quantified by Trypan blue exclusion assay.

Influenza virus replication. MDCK cells were infected with various strains of influenza virus depicted in the figures at an m.o.i. of 0.001 p.f.u. per cell for $1 \mathrm{~h}$. Next, cells were washed with PBS and overlaid with OptiMEM containing twofold compound dilutions ranging from $100 \mu \mathrm{M}$ to $0.8 \mu \mathrm{M}$. Samples containing only the same volume of DMSO as the compounds were included. At $30 \mathrm{~h}$ after infection, culture medium was collected, and cell debris was removed by centrifugation at $1,000 \mathrm{~g}$ for $10 \mathrm{~min}$ and frozen at $-80^{\circ} \mathrm{C}$. Viral titers were determined by plaque assay. The experiments conducted with the H1N1/1918 strain were performed in a high-containment (BSL3++) facility.

For experiments performed with U20S cells, cells were plated in 12-well plates in DMEM containing 10\% (v/v) FBS and incubated overnight. Cells were then incubated in medium containing tetracycline $\left(1 \mu \mathrm{g} \mathrm{ml}^{-1}\right)$ for $2 \mathrm{~h}$ to induce REDD1 overexpression. Cells were washed with PBS and infected with A/WSN/1933 or VSV at m.o.i. 2 for $1 \mathrm{~h}$. Tetracycline was again added $1 \mathrm{~h}$ after infection, and cell lysates were prepared at various time points after infection, as indicated in Figure 6.

VSV replication assay. VSV replication: MDCK cells seeded in $35 \mathrm{~mm}$-diameter dishes were infected with VSV-GFP at m.o.i. 0.001 p.f.u. per cell. At $24 \mathrm{~h}$ after infection, supernatants were clarified and used for titration on Vero cells. Fourfold serial dilutions of virus containing supernatants were made in PBS containing serum and antibiotics. Fifty microliters of each dilution were mixed with an equal volume of complete growth medium containing 8,000 Vero cells and incubated at $37^{\circ} \mathrm{C}$ for $48 \mathrm{~h}$ in 96 -well plates. Cells were fixed in $4 \%(\mathrm{v} / \mathrm{v})$ paraformaldehyde. The number of wells with GFP expression were counted by fluorescence microscopy and subsequently used to calculate relative virus titers. Infection of U2OS cells with VSV was performed in the same manner as influenza virus infection described above.

In situ hybridization. mRNA distribution in MDCK cells infected with influenza virus in the presence or absence of compounds was performed as previously described ${ }^{18}$. Influenza proteins were detected with mouse influenza-specific antibody (Biodesign International) and FITC-labeled mouse-specific antibody (Invitrogen).
Phospho-S6K analysis. Cells were starved for $18 \mathrm{~h}$ and then mock infected or infected as described in the legend of Figure 5. Five percent serum was added to induce S6K phosphorylation in control lanes. H358 and H1993 cells were treated with $10 \mu \mathrm{M} 3$, and LnCap cells were treated with $30 \mu \mathrm{M}$.

All data presented here are representative of at least three independent experiments. In the line graphs or histograms, data represent mean values \pm s.d.

Description of real-time PCR, gene expression profiling and analysis, human biochemical network, compound synthesis, details of cells, plasmids and antibodies are described in Supplementary Methods.

\section{Received 20 May 2011; accepted 1 July 2011;} published online 11 September 2011

\section{References}

1. Simonsen, L. et al. The impact of influenza epidemics on mortality: introducing a severity index. Am. J. Public Health 87, 1944-1950 (1997).

2. Smith, D.J. et al. Mapping the antigenic and genetic evolution of influenza virus. Science 305, 371-376 (2004).

3. Krug, R.M. \& Etkind, P.R. Cytoplasmic and nuclear virus-specific proteins in influenza virus-infected MDCK cells. Virology 56, 334-348 (1973).

4. Li, Y., Yamakita, Y. \& Krug, R.M. Regulation of a nuclear export signal by an adjacent inhibitory sequence: the effector domain of the influenza virus NS1 protein. Proc. Natl. Acad. Sci. USA 95, 4864-4869 (1998).

5. Guo, Z. et al. NS1 protein of influenza A virus inhibits the function of intracytoplasmic pathogen sensor, RIG-I. Am. J. Respir. Cell. Mol. Biol. 36, 263-269 (2007).

6. Mibayashi, M. et al. Inhibition of retinoic acid-inducible gene I-mediated induction of beta interferon by the NS1 protein of influenza A virus. J. Virol. 81, 514-524 (2007).

7. Opitz, B. et al. IFNbeta induction by influenza A virus is mediated by RIG-I which is regulated by the viral NS1 protein. Cell Microbiol. 9, 930-938 (2007)

8. Pichlmair, A. et al. RIG-I-mediated antiviral responses to single-stranded RNA bearing 5'-phosphates. Science 314, 997-1001 (2006).

9. Li, S., Min, J.Y., Krug, R.M. \& Sen, G.C. Binding of the influenza A virus NS1 protein to PKR mediates the inhibition of its activation by either PACT or double-stranded RNA. Virology 349, 13-21 (2006).

10. Min, J.Y. \& Krug, R.M. The primary function of RNA binding by the influenza A virus NS1 protein in infected cells: Inhibiting the $2^{\prime}-5^{\prime}$ oligo (A) synthetase/RNase L pathway. Proc. Natl. Acad. Sci. USA 103, 7100-7105 (2006).

11. Hale, B.G., Jackson, D., Chen, Y.H., Lamb, R.A. \& Randall, R.E. Influenza A virus NS1 protein binds p85beta and activates phosphatidylinositol-3-kinase signaling. Proc. Natl. Acad. Sci. USA 103, $14194-14199$ (2006).

12. Fortes, P., Beloso, A. \& Ortin, J. Influenza virus NS1 protein inhibits pre-mRNA splicing and blocks mRNA nucleocytoplasmic transport. $E M B O J$ 13, 704-712 (1994)

13. Qiu, Y., Nemeroff, M. \& Krug, R.M. The influenza virus NS1 protein binds to a specific region in human U6 snRNA and inhibits U6-U2 and U6-U4 snRNA interactions during splicing. RNA 1, 304-316 (1995).

14. Wolff, T., O’Neil, R.E. \& Palese, P. NS1-Binding protein (NS1-BP): a novel human protein that interacts with the influenza A virus nonstructural NS1 protein is relocalized in the nuclei of infected cells. J. Virol. 72, 7170-7180 (1998).

15. Nemeroff, M.E., Barabino, S.M., Li, Y., Keller, W. \& Krug, R.M. Influenza virus NS1 protein interacts with the cellular $30 \mathrm{kDa}$ subunit of CPSF and inhibits 3' end formation of cellular pre-mRNAs. Mol. Cell 1, 991-1000 (1998).

16. Qian, X.-Y., Alonso-Caplen, F. \& Krug, R.M. Two functional domains of influenza virus NS1 protein are required for regulation of nuclear export of mRNa. J. Virol. 68, 2433-2441 (1994).

17. Qiu, Y. \& Krug, R.M. The influenza virus NS1 protein is a poly(A)-binding protein that inhibits nuclear export of mRNAs containing poly(A). J. Virol. 68, 2425-2432 (1994).

18. Satterly, N. et al. Influenza virus targets the mRNA export machinery and the nuclear pore complex. Proc. Natl. Acad. Sci. USA 104, 1853-1858 (2007).

19. García-Sastre, A. et al. Influenza A virus lacking the NS1 gene replicates in interferon-deficient systems. Virology 252, 324-330 (1998).

20. Brugarolas, J. et al. Regulation of mTOR function in response to hypoxia by REDD1 and the TSC1/TSC2 tumor suppressor complex. Genes Dev. 18, 2893-2904 (2004).

21. Ma, X.M. \& Blenis, J. Molecular mechanisms of mTOR-mediated translational control. Nat. Rev. Mol. Cell. Biol. 10, 307-318 (2009)

22. Chakraborty, P. et al. Nucleoporin levels regulate cell cycle progression and phase-specific gene expression. Dev. Cell 15, 657-667 (2008).

23. Tumpey, T.M. et al. Characterization of the reconstructed 1918 Spanish influenza pandemic virus. Science 310, 77-80 (2005).

24. Hresko, R.C. \& Mueckler, M. mTOR.RICTOR is the Ser ${ }^{473}$ kinase for Akt/ protein kinase B in 3T3-L1 adipocytes. J. Biol. Chem. 280, 40406-40416 (2005). 
25. Sarbassov, D.D., Guertin, D.A., Ali, S.M. \& Sabatini, D.M. Phosphorylation and regulation of Akt/PKB by the rictor-mTOR complex. Science 307, 1098-1101 (2005).

26. Rubinsztein, D.C., Gestwicki, J.E., Murphy, L.O. \& Klionsky, D.J. Potential therapeutic applications of autophagy. Nat. Rev. Drug Discov. 6, 304-312 (2007).

27. Vega-Rubin-de-Celis, S. et al. Structural analysis and functional implications of the negative mTORC1 regulator REDD1. Biochemistry 49, 2491-2501 (2010).

28. Buchkovich, N.J., Yu, Y., Zampieri, C.A. \& Alwine, J.C. The TORrid affairs of viruses: effects of mammalian DNA viruses on the PI3K-Akt-mTOR signalling pathway. Nat. Rev. Microbiol. 6, 266-275 (2008).

29. Cooray, S. The pivotal role of phosphatidylinositol 3-kinase-Akt signal transduction in virus survival. J. Gen. Virol. 85, 1065-1076 (2004).

30. Ehrhardt, C. et al. Influenza A virus NS1 protein activates the PI3K/Akt pathway to mediate antiapoptotic signaling responses. J. Virol. 81, 3058-3067 (2007).

31. Hale, B.G., Jackson, D., Chen, Y.H., Lamb, R.A. \& Randall, R.E. Influenza A virus NS1 protein binds p85beta and activates phosphatidylinositol-3-kinase signaling. Proc. Natl. Acad. Sci. U.S.A. 103, 14194-14199 (2006).

32. Shin, Y.K., Liu, Q., Tikoo, S.K., Babiuk, L.A. \& Zhou, Y. Influenza A virus NS protein activates the phosphatidylinositol 3-kinase (PI3K)/Akt pathway by direct interaction with the p85 subunit of PI3K. J. Gen. Virol. 88, 13-18 (2007)

33. Zhirnov, O.P. \& Klenk, H.D. Control of apoptosis in influenza virus-infected cells by up-regulation of Akt and p53 signaling. Apoptosis 12, 1419-1432 (2007).

34. Konig, R. et al. Human host factors required for influenza virus replication. Nature 463, 813-817 (2010).

35. Brugarolas, J. mTORC1 signaling and hypoxia. in mTOR Pathway and mTOR Inhibitors in Cancer Therapy (eds. Polunovsky, V. \& Houghton, P.J.) 75-79 (Humana Press, 2010).

36. Todd, D.J., Lee, A.H. \& Glimcher, L.H. The endoplasmic reticulum stress response in immunity and autoimmunity. Nat. Rev. Immunol. 8, 663-674 (2008).

37. Zinkernagel, A.S., Johnson, R.S. \& Nizet, V. Hypoxia inducible factor (HIF) function in innate immunity and infection. J. Mol. Med. 85, 1339-1346 (2007).

38. Krishnamoorthy, J., Mounir, Z., Raven, J.F. \& Koromilas, A.E. The eIF2alpha kinases inhibit vesicular stomatitis virus replication independently of eIF2alpha phosphorylation. Cell Cycle 7, 2346-2351 (2008)

39. Jin, H.-O. et al. Activating transcription factor 4 and CCAAT/enhancerbinding protein- $\beta$ negatively regulate the mammalian target of rapamycin via Redd 1 expression in response to oxidative and endoplasmic reticulum stress. Free Radic. Biol. Med. 46, 1158-1167 (2009).

40. Whitney, M.L., Jefferson, L.S. \& Kimball, S.R. ATF4 is necessary and sufficient for ER stress-induced upregulation of REDD1 expression. Biochem. Biophys. Res. Commun. 379, 451-455 (2009)

41. Costa-Mattioli, M., Sossin, W.S., Klann, E. \& Sonenberg, N. Translational control of long-lasting synaptic plasticity and memory. Neuron 61, 10-26 (2009).

42. Shahbazian, D. et al. Control of cell survival and proliferation by mammalian eukaryotic initiation factor 4B. Mol. Cell. Biol. 30, 1478-1485 (2010).
43. Farny, N.G., Hurt, J.A. \& Silver, P.A. Definition of global and transcriptspecific mRNA export pathways in metazoans. Genes Dev. 22, 66-78 (2008).

44. Keene, J.D. RNA regulons: coordination of post-transcriptional events. Nat. Rev. Genet. 8, 533-543 (2007)

45. Kao, R.Y. et al. Identification of influenza A nucleoprotein as an antiviral target. Nat. Biotechnol. 28, 600-605 (2010).

46. Hoffmann, H.H., Kunz, A., Simon, V.A., Palese, P. \& Shaw, M.L. Broadspectrum antiviral that interferes with de novo pyrimidine biosynthesis. Proc. Natl. Acad. Sci. USA 108, 5777-5782 (2011).

47. Choo, A.Y. \& Blenis, J. Not all substrates are treated equally: implications for mTOR, rapamycin-resistance and cancer therapy. Cell Cycle 8, 567-572 (2009).

48. McNaney, C.A. et al. An automated liquid chromatography-mass spectrometry process to determine metabolic stability half-life and intrinsic clearance of drug candidates by substrate depletion. Assay Drug Dev. Technol. 6, 121-129 (2008).

\section{Acknowledgments}

We thank R. Sakthivel (University of Texas Southwestern) for technical assistance with plaque assays and L. Melito and J. Naidoo (University of Texas Southwestern) for technical assistance with compound synthesis. We thank S. Rubin de Celis (University of Texas Southwestern) for providing REDD1 cells, D. Levy (New York University) for providing Stat $1^{-/}$cells and B. Levine (University of Texas Southwestern) for providing $A T G 5^{-/}$cells and LC3 antibodies. This work was supported by US National Institutes of Health (NIH) grants R01GM07159 to B.M.A.F.; R01AI079110 and R01AI089539 to B.M.A.F. and M.G.R.; the Diane and Hal Brierley Distinguished Chair in Biomedical Research to M.G.R; National Center for Research Resources grant C06-RR15437; NIH grants R01AI046954, P01AI058113, U54AI057158 and U01AI074539; the Center for Research on Influenza Pathogenesis, the National Institute of Allergy and Infectious Diseases-funded Center of Excellence for Influenza Research and Surveillance (HHSN266200700010C to A.G.-S.); NIH grant R01CA129387 to J.B.; and support to M.A.M. from the NIH Diversity Supplement (R01GM06715908S1).

\section{Author contributions}

M.A.M., N.S., G.A.V., D.F., S.P.-L., J.B., C.F., M.A.W., A.G.-S., M.G.R. and B.M.A.F. designed the research; M.A.M., N.S., G.A.V., S.W., N.W., M.S., S.P.-L. and C.F. performed the research; D.F. contributed new reagents; M.A.M., N.S., G.A.V., D.F., N.W., M.S., S.P.-L., J.B., C.F., M.A.W., A.G.-S., M.G.R. and B.M.A.F analyzed data; M.G.R. and B.M.A.F. wrote the paper.

\section{Competing financial interests}

The authors declare no competing financial interests.

\section{Additional information}

Supplementary information, chemical compound information and chemical probe information is available online at http://www.nature.com/naturechemicalbiology/. Reprint and permissions information is available online at http://www.nature.com/reprints/index. html. Correspondence and requests for materials should be addressed to B.M.A.F. 